\title{
ALGUNOS PINTORES Y ESCULTORES DE LA GIUDAD EN EL SIGLO XVIII
}

(Según padrones del Sagrario Metropolitano)

Por Salvador Cruz.

A don Manuel Romero de Terreros.

Durante la Colonia, el Sagrario fue la parroquia de españoles más importante en la ciudad de México. Igual primacía se apreciaba en las restantes metrópolis y ciudades catedralicias de la América hispánica.

El máximo esplendor de la feligresía central de México en el siglo xviII, podemos reconstruirlo con meridiana seguridad a través de los Padrones de Cumplimiento, que anualmente se levantaban para servicio de la iglesia.

Seis de estos padrones, pertenecientes a una biblioteca particular, nos han sido franqueados. ${ }^{1 \cdot 6}$ Corresponden a los años de 1737, 1753, 1759,

1 Padrón de este año de 1737 perteneciente al S. D. D. Ioseph Ramires de el Castillo Cura Propietario de el Sagrario de esta Sancta Iglecia Cathedral de México, \&c... Br. Joseph Valadez lo escriuio.-1 vol. manuscrito. Portada coloreada. Pasta de pergamino. Sin foliar.

2 Padrón que pertenese al Señor Lizenciado Don Joachín López del Barrio Cura más antiguo de esta Santa Yglesia Cathedral de México; Año de el Sr. de 1753.-1 vol. manuscrito. Pasta de pergamino. Foliado: $113 \mathrm{ff}$.

3 Padrón del año del Señor de 1759. Perteneciente al Señor Licenciado D. Ignacio Carrillo Benitúa, Cura por su Mag. de el Sagrario de esta Santa Iglesia Cathedral de México, \&c. -1 vol. manuscrito. Portada impresa. Pasta de piel con impresiones doradas. Sin foliar.

4 Padrón que para el cumplimiento de N.S.M. Iglecia, hizo S.L.D. Carlos Celedonio de Velásquez, como Cura del Sagrario de dicha Santa Yglecia, comensado en quatro de Marzo del año de 1767, \&c.-1 vol. manuscrito. Pasta de piel con impresiones doradas. Sin foliar.

5 Padrón... de Orden de los Señores Curas de el Sagrario de esta Santa Iglesia, en este presente año de mil setecientos setenta y cinco años. 1 vol. manuscrito. Portada con una acuarelita en la parte superior. Pasta de piel, manchada artificialmente, con impresiones doradas. Broches originales de cuentas de cristal azul. Sin foliar.

6 Padrón y Ramo del Cumplimiento Anual de Nuestra Santa Madre Yglesia, el que corresponde a el Sagrario de esta Santa Yglesia Cathedral de México... Año del Señor de 1775.-1 vol. manuscrito. Portada de la misma mano que la anterior, 
1767 y dos de 1775 (a y b). Afortunadamente cada uno contiene Ramos diferentes - secciones, diriamos hoy-, divididos en cuadros - manzanas-, con expresión de aceras, calles, callejones, rinconadas, etcétera. Los cuadros resultaban ser la unidad estructural de la feligresía -una de las cuatro que hacían al México colonial en lo eclesiástico.

De tan valioso acervo manuscrito hemos querido extraer los nombres de los pintores y los escultores. A unos, claramente se les menciona en ejercicio; a otros, se les identifica por sus relaciones de familia; a un tercer grupo, sujeto a ratificación, no lo hemos querido dejar de anotar con la seguridad de que abren campo a futuras investigaciones.

Por ahora, han quedado revisados poco más o menos cuarenta y cinco mil nombres.

\section{7}

Calle Serada de Sta. Ynes. [Cerrada de Santa Inés: $1^{\text {a }}$ de la Academia.] $Q$ (uart) $O$-Dn. Miguel Cabrera, ? D. Joseph Serbantes (Contigua, casa de) El Sr. Br. Dn. Joseph Telles Xiron, Con 4 de familia.

Calle Chabaria. [Chavarría: 2a de Justo Sierra.]

Cassa de Dn. Joseph Alsibar ${ }^{8}$ con cinco.

Calle de S. P. y S. Pablo. [San Pedro y San Pablo: $2^{a}$ de San Ildefonso.] A (ccesori) a - M (aest) ro Joseph de Coria, ${ }^{9}$ Josepha Seceña y Antonio Coria, Miguel Pintor.

\section{3}

Sera de enfrente del Arquillo que bue(l)be para la Casuela. [Desapareció al ampliarse la Avenida del 5 de Mayo.]

con una acuarelita en la parte superior, muy interesante. Pasta de piel, con impresiones doradas. Sin foliar.

7 En 1737, Cabrera estaba soltero; se casó tres años después con doña Ana María Solano de Herrera. Como sucede con otros pintores de la presente nómina, se hacia constar el nombre del ayudante allegado; aquí José Cervantes parece serlo. Cabrera, que siempre perteneció a la feligresía del Sagrario, se encuentra sepultado en Santa Inés.

$8 \mathrm{La}$ familia de José de Alcíbar siempre perteneció al Sagrario, como lo atestigua la constante aparición del apellido en otros padrones. Se sabía que Cabrera fue maestro de Alcibar pero ahora parece que eran también vecinos.

- ¿Este Maestro José de Coria, marido de Josefa Ceceña y con un hijo Antonio, no tendrá mucho que ver, por la fecha inclusive, con el primer Cora, José Antonio Villegas de Cora? Un pintor Miguel, sin duda indigena, los acompaña. Preciosa incógnita para futuras investigaciones. 
Cassa alta - Juan Patricio (Morlete) Ruiz, ${ }^{10}$ María Josepha Cariaga, María de Jesús, María Antonia, Maria Máxima Rozalía, Ramón.

De la plazuela de Jesús para San Agustin. [República de El Salvador, entre 20 de Noviembre y 5 de Febrero.]

147. Asesor (i) a. Pintor Manuel Guido, ${ }^{11}$ Ana Benjumea, Blas Guido. Espaldas del Hospital de Jesús. [Calle cerrada de Jesús. Hoy, ampliada, es Av. 20 de Noviembre, entre Rep. de El Salvador y Mesones.]

234. A (cseroi) a del $M$ (aes) tro Pintor Manuel Musiño ${ }^{12}$ con 5. Calle del Parque. [Hoy Rep. de El Salvador, entre Pino Suárez y Correo Mayor.]

625. A (csesori) a del Pintor Manuel Basquez, ${ }^{13}$ Matías Huerta. Cera del Amor de Dios. [2a de Academia.]

766. A (csesori) a del Pintor Juan Antonio Arriaga ${ }^{14}$ con 7. Calle de las Moscas. [Jesús María, entre Emiliano Zapata y Soledad?] 806. A (csesori) a Pintor Juan Franco, ${ }^{15}$ Ana González Joseph Belasco. Calle de la Merced, para el Volador y la Universidad. [Venustiano Carranza, entre Correo Mayor y Jesús María.]

1113. A (csesori) a del Pintor Antonio (Pérez de?) Aguilar, ${ }^{16}$ Manuela Aguilar, Josepha Muñoz.

\section{7}

Callejón del Arquillo de la Profesa. [Desapareció al ampliarse la Avenida del 5 de Mayo.]

10 Morlete Ruiz también fue feligrés constante del Sagrario, con casa en el callejón del Arquillo. (Vid. nota 17.)

11 Nótese que este pintor y los subsecuentes mencionados en el padrón de 1759, tenían sus talleres localizados en una pequeña zona, de la cual surgiría, veintidós años más tarde, el pie fundador de la Real Academia de Nobles Artes de San Carlos de la Nueva España.

12 Idem.

13 Ibid.

14 Ibid.

15 Ibid.

10 Ibid. ¿Será éste el "casi desconocido" pintor Antonio Pérez de Aguilar? 
Casa Alta. Arriba.-Dn. Juan Patricio de Morlete (Ruiz), ${ }^{17}$ Da. María Josepha (Careaga) Medina, María de Jesús Morlete, María Michaela, María Josepha, María Ignacia, María Andrea, Maria Isabel, Francisca, Joseph, Joseph Antonio, Francisco, Martín Bisente.

Calle de las Medinas. [República de Cuba, entre Brasil y Palma.]

Asesoria. Escultor Santiago Sandobal, ${ }^{18}$ Rita Sisneros, María Francisa, Ignacia Sandobal, Joseph Saloma.

Callejón de los Melemitas (sic por, Bethlemitas). [1"a de Filomeno Mata.]

N. 13. A (csesori) a Joseph Pais ${ }^{19}$ su esp (os) a, Anna Morales (contigua) Casa Grande del Sr. Conde de Santiago \&c.

Calle de Manrique. [República de Chile, entre Tacuba y Donceles.]

(Acsesoria) sin $\mathbf{n}$ (úmer) o, Pintorería de Antonio Sánchez, ${ }^{20}$ Margarita Garcia con 3.

(En este mismo padrón se cita otra Pintorería y dos Obradores de Pintor, cuyos dueños no dormían ahí y por lo tanto no se hacen constar sus nombres.)

\section{$1775 \mathrm{~b}$}

Calle de la Pila Seca. [Había $1^{\mathrm{a}}$ y $2^{\mathrm{a}}$ de la Pila Seca, hoy $3^{\mathrm{a}}$ y $4^{\mathrm{a}}$ de Chile.] Vivienda Prinsipal de Dn. Joseph Pais, ${ }^{21}$ con cinco, y d (ic) ho.

17 Se comprueba la numerosa descendencia de Morlete Ruiz y ahora sabemos el segundo apellido de su esposa.

18 único escultor citado como tal: Santiago Sandoval, marido de Rita Cisneros con una hija, Ignacia. Dos adultos: María Francisca, sin duda indígena y Joseph Saloma, probablemente ayudante.

19 Queda sujeto a ratificación este persanaje que pudiera corresponder a don José de Páez. (Vid. nota 21.)

$20 \mathrm{Al}$ hacerse la distinción de Pintorería y Obrador de pintor, pudiera ser que lo primero correspondiera a nuestros actuales expendios de pinturas y los obradores no sean sino el claro equivalente del taller.

24 Vale lo dicho en la nota 19, que corresponde al mismo año de 1775. 